\title{
CARACTERIZACIÓN DE LOS AGROECOSISTEMAS CON PRODUCCIÓN OVINA EN EL ORIENTE DE YUCATÁN, MÉXICO ${ }^{1}$
}

\author{
Bernardino Candelaria-Martínez ${ }^{2}$, Carolina Flota-Bañuelos ${ }^{2}$, Luis Enrique Castillo-Sánchez ${ }^{3}$
}

\section{RESUMEN}

Caracterización de los agroecosistemas con producción ovina en el oriente de Yucatán, México. El objetivo de este estudio fue caracterizar y agrupar los agroecosistemas con producción ovina de la región oriente de Yucatán, México. Este estudio se realizó de agosto 2012 a abril 2013. Se aplicó un cuestionario semiestructurado mediante entrevista al $93 \%$ de los productores de siete municipios, que manejaban ovinos dentro de sus agroecosistemas. Se realizó un muestreo aleatorio, análisis de conglomerados, ANOVA mediante GLM y comparación de medias por Tukey $(\mathrm{P}>0,05)$. Se obtuvieron cuatro grupos diferentes de agroecosistemas con ovinos: GA (responsables con edad avanzada, escolaridad media, alta cantidad de tierras, rebaños grandes y más años de practicar la ovinocultura), GB (responsables de edad intermedia, mayor escolaridad y cantidad intermedia de tierras, tamaño intermedio de rebaño), GC (responsables de edad avanzada, menor escolaridad, baja cantidad de tierras y reducido tamaño del rebaño) y GD (responsables más jóvenes, escolaridad alta, baja cantidad de tierras, rebaño pequeño y menor tiempo de realizar la actividad). La ovinocultura fue la primera, segunda y tercera opción productiva para el 12, 75 y 11,8\% de los productores, respectivamente. En la región, la ovinocultura es una actividad practicada desde hace más de quince años en algunos casos, sin embargo, solamente es la prioridad económica de un pequeño grupo de productores. El acceso a recursos determinó su grado de desarrollo y fue evidente la falta de organización entre los ovinocultores.

Palabras clave: empresas agrarias, ovinocultura, pequeños rumiantes.

\begin{abstract}
Characterization of agroecosystems with sheep production in the eastern side of Yucatán, Mexico. The aim of this study was to characterize and group agroecosystems with sheep production in the eastearn side of Yucatan, Mexico. This study was held from August 2012 to April 2013. A semi-structured questionnaire was applied while interviewing $93 \%$ of producers from seven municipalities that have sheep within their agroecosystems. Random sampling, cluster analysis, ANOVA using GLM and comparison of means by Tukey $(\mathrm{P}>0.05)$ were performed. Four different groups of agroecosystems with sheep production were obtained: GA (elderly people in charge, middle schooling, and high amount of land, large herds or more years practicing sheep farming), GB middle age people in charge, higher schooling, intermediate amount of land, and intermediate herd size), GC (elderly people in charge, less schooling, low amount of land and reduced herd size), and GD (younger people in charge, high schooling, low amount of land, small herd size and less time performing the activity). Sheep farming was the first, second, and third productive option for $12 \%, 75 \%$ and $11,8 \%$ of producers. In some cases, sheep production in the region has been practiced for over 15 years; however it is the economic priority only of a small group of producers. Access to resources determined their level of development and lack of organization among sheep producers is evident.
\end{abstract}

Keywords: farms, producers, production sheep, small ruminants.

\footnotetext{
Recibido: 11 de diciembre, 2014. Aceptado: 10 de febrero, 2015. Proyecto financiado por la Dirección General de Educación Superior Tecnológica. Clave del proyecto DGEST 4619.12-P.

2 Colegio de Postgraduados, Campus Campeche. Carretera Haltunchén-Edzna, km 17.5, Sihochac, Champotón, Campeche. México. CP. 24450.cmartinez@colpos.mx, cflota@colpos.mx (autor para correspondencia).

3 Instituto Tecnológico de Tizimín. Final aeropuerto cupul S/N, Tizimín, Yucatán, México. hymenopteralecs@hotmail.com
} 


\section{INTRODUCCIÓN}

La cría de ovinos en las regiones tropicales de México se percibe, actualmente, como una opción ganadera con alto potencial de desarrollo (Morales et al., 2004), originado por una aceptación creciente por parte de los consumidores (Martínez-Partida et al., 2011); quienes gradualmente incluyen la carne ovina en su dieta (Arteaga, 2007), con un consumo per cápita de 1,1 kg de carne ovina (SIAP, 2012). En 2013, se tuvo una producción nacional de $113342 \mathrm{t}$ de carne ovina en pie, equivalente a 57,980 t de carne en canal, originada por el sacrificio de 2915534 ovinos (SIAP, 2014). Asimismo, la FAO (2013) prevé un incremento del consumo mundial de carne ovina en los próximos años.

En México existen regiones con potencial para el desarrollo de sistemas de manejo que resulten amigables con el ambiente y con elevados índices de producción, haciendo uso óptimo de los recursos naturales disponibles. El mercado de la carne de ovinos de pelo se centra principalmente en los Estados del centro del país, en donde existe un elevado número de intermediarios y detallistas con amplia capacidad de absorción de la producción nacional (Partida et al., 2013; Morales et al., 2004).

En el año 2013, Yucatán ocupó el lugar dieciocho a nivel nacional en producción de carne ovina en canal, con una cifra de $1606 \mathrm{t}$ de carne en pie, $811 \mathrm{t}$ de carne en canal por el sacrificio de 44824 ovinos (SIAP, 2013), y aportó el 1,29\% del inventario del rebaño nacional. La región oriente del estado de Yucatán presenta una marcada vocación a la ganadería, que durante mucho tiempo ha estado dominada por la producción bovina; sin embargo, en los últimos diez años se ha observado una tendencia hacia la producción ovina, motivada especialmente por factores como la reducción de la capacidad de carga animal en las praderas ganaderas, la estabilidad del precio del cordero en el mercado y el incremento de la demanda de ovinos en desarrollo o finalizados por compradores del centro del país y por procesadores regionales, ubicados en la Riviera Maya, donde existe una alta demanda por parte del sector de restaurantes. No obstante, cada vez son mayores las exigencias del mercado en calidad y homogeneidad de los subproductos ovinos (Sañudo, 2008), por lo que es necesario implementar programas de mejoramiento de la genética de los rebaños, manejo sanitario, reproductivo y de alimentación, a fin de incrementar la calidad (Frías et al., 2011).
La participación gubernamental ha sido importante en los últimos años, destinando recursos para el fomento de la ovinocultura. Sin embargo, es poco perceptible el efecto de estas acciones, debido a que no se tiene información que precise el estado y características que guardan los sistemas de producción ovina regionales, para tomarse como punto de partida en el diseño y la gestión de programas encaminados a fomentar la ovinocultura, posicionándola como una actividad ambiental y económicamente viable, capaz de apuntalar el desarrollo regional. En este sentido, el enfoque de agroecosistemas (AES) permite dimensionar de manera integral la estructura, funcionamiento e interrelaciones internas y externas de los sistemas de producción agropecuaria, lo cual facilita la toma de decisiones y permite generar conocimiento aplicable bajo diferentes contextos (Gallardo et al., 2002; Vilaboa et al., 2009).

Con base a lo anterior, el objetivo de este estudio fue caracterizar y agrupar los agroecosistemas con producción ovina de la región oriente de Yucatán, México.

\section{MATERIALES Y MÉTODOS}

El estudio se realizó de agosto de 2012 a abril de 2013, en siete municipios de la región oriente del estado de Yucatán: Espita, Panabá, San Felipe, Sucilá, Tizimín, Temozón y Valladolid, ubicados entre $20^{\circ} 41^{\prime} 06^{\prime \prime} \mathrm{N}, 88^{\circ} 12^{\prime} 00^{\prime \prime} \mathrm{W}$ y $20^{\circ} 59^{\prime} 13$ ” $\mathrm{N}$ y $87^{\circ} 33^{\prime} 16^{\prime \prime} \mathrm{W}$.

Para determinar el tamaño muestral, se utilizó un diseño de muestreo aleatorio. Se tomó una muestra de $93 \%$, correspondiendo a 161 productores de la población total de los agroecosistemas con producción ovina de los siete municipios. Siendo la población total 173 agroecosistemas (INEGI, 2013) (Cuadro 1).

Se diseñó un cuestionario semiestructurado con ochenta preguntas agrupadas en seis secciones: información general, infraestructura, manejo reproductivo, sanitario, de alimentación, de las praderas y comercialización, el cual se aplicó mediante entrevistas directas a los responsables de los sistemas de producción ovina de los siete municipios.

Posterior a la aplicación del cuestionario, se realizó un recorrido por los 173 agroecosistemas, donde se observaron las instalaciones y se registró la memoria fotográfica. Los datos de los cuestionarios se incluyeron en hojas del programa Microsoft Office 
Cuadro 1. Relación de agroecosistemas con ovinos muestreados por municipio en el oriente del estado de Yucatán, México. 2013. Table 1. Relation of agroecosystems with sheep sampled by the municipality in the eastern side of Yucatan, Mexico. 2013.

\begin{tabular}{lcccc}
\hline Municipio & $\begin{array}{c}\text { Número de unidades } \\
\text { de producción* }\end{array}$ & Porcentaje & $\begin{array}{c}\text { Productores } \\
\text { encuestados }\end{array}$ & Porcentaje \\
\hline Espita & 8 & 4,62 & 31 & 19,25 \\
Panabá & 26 & 15,03 & 28 & 17,39 \\
San Felipe & 8 & 4,63 & 5 & 3,11 \\
Sucilá & 36 & 20,81 & 30 & 18,64 \\
Tizimín & 72 & 41,62 & 43 & 26,71 \\
Temozón & 14 & 8,09 & 20 & 12,42 \\
Valladolid & 9,20 & 4 & 2,48 \\
Total & 173 & 100 & 161 & 100 \\
\hline
\end{tabular}

* Fuente: INEGI (2013) / Source: INEGI (2013).

Excel 2007, posteriormente se codificaron y clasificaron en respuestas abiertas y cerradas, a las cerradas se les ponderó valores si $=1$ y no=0. Distinguiendo doce variables: edad del productor (años), escolaridad del productor (años), extensión del agroecosistema (ha), tipo de tenencia de la tierra (propiedad privada, ejidal o comunal), importancia de la ovinocultura en el agroecosistema (primera, segunda o tercera actividad), tamaño del rebaño (cabezas), años dedicados a la ovinocultura (años), acceso a asistencia técnica ( $\mathrm{si}=0$, no $=0$ ), organización con otros productores $(\mathrm{si}=0$, no $=0)$, acceso a financiamiento $(\mathrm{si}=0, \mathrm{no}=0)$, acceso a apoyos de gobierno para la actividad $(\mathrm{si}=0$, no $=0)$ y disponibilidad para contratar los servicios de un técnico $(\mathrm{si}=0$, no=0). Se realizó un análisis descriptivo de las variables de homogeneidad, análisis de correlación y tipificación de los conglomerados por vinculación completa (Santesmases, 2005), utilizando distancias euclidianas y el algoritmo de ligamiento simple o vecino más cercano (Ojeda, 1999) con el programa estadístico SPSS Statistics (2006).

A las variables usadas para el análisis por conglomerados, aunado a variables relacionadas con el nivel tecnológico y manejo de los recursos (uso de corrales y mangas de manejo, uso de corrales de maternidad, tipo de mano de obra (contratada o familiar), tipo de empadre, relación hembra:macho, asistencia de partos, uso de suplemento alimenticio, uso de sistemas silvopastoriles y comercialización); se les aplicó un análisis de varianza y pruebas de medias para determinar diferencias estadísticas entre grupos mediante Tukey $(\mathrm{P}<0,05)$.

\section{RESULTADOS Y DISCUSIÓN}

\section{Importancia y generalidades de la producción ovina del oriente de Yucatán}

Se observó que en los agroecosistemas con ovinos de la región oriente de Yucatán, la ovinocultura ocupó el segundo lugar en orden de importancia económica para el $75,1 \%$ de los responsables encuestados; mientras que para el $12,4 \%$ y $11,8 \%$ representó la primera o tercera opción de importancia económica, respectivamente. En el 61,5\% de los casos, la producción de ovinos se desarrolló paralelamente a la cría de bovinos, dichos resultados son inferiores a los reportados por Morales et al. (2004) en un ejido del estado de Veracruz, en donde mencionaron que el $93 \%$ de los productores de ovinos también criaban ganado bovino. Por otro lado, los resultados de este estudio coinciden con lo reportado por Pérez et al. (2011) quienes encontraron que el $66 \%$ de los productores asoció la cría de ovinos con la producción de ganado bovino. El 56\% de los productores entrevistados mencionó que la producción de ovinos en sus agroecosistemas es discontinua, y que la decisión del periodo de cría y la cantidad de 
animales manejados se encuentra en función de la disponibilidad de alimento en la unidad de producción, en donde se intenta satisfacer en primera instancia las demandas de alimento del ganado bovino.

En cuanto al tamaño de los agroecosistemas, se observó una extensión promedio de 33,57 ha, con valores mínimos de 0,1 y máximos de 400 ha. Estos valores muestran una amplia heterogeneidad en la disponibilidad de tierras por parte de los productores que manejan ovinos, desde manejo de traspatio, hasta grandes extensiones. Este fenómeno ya había sido reportado por Góngora-Pérez et al. (2010), quienes mencionaron extensiones de una a más de 30 ha para ovinocultores del estado de Yucatán, sin especificar el valor máximo. Fue posible apreciar que la cría de ovinos representa una práctica adoptada por productores de diferentes estratos, y constituye una opción de ahorro familiar desde la estructura de la ganadería de traspatio (Morales et al., 2004), una actividad empresarial prometedora (Pérez et al., 2011) o una actividad de esparcimiento para profesionales pensionados o activos.

La edad promedio de los productores fue de 50,8 años con rangos de 17 hasta 80 años; mostrando que la edad no interfirió en la realización de la ovinocultura, lo que resultó interesante, considerando que los productores agropecuarios suelen presentar resistencia al cambio (Suárez et al., 2012), especialmente los de mayor edad (Córdova, 1993). Por otra parte, el incremento de la demanda de carne ovina (Pérez et al., 2011) y un precio más competitivo en el mercado comparado con otras especies ganaderas (GóngoraPérez et al., 2010), han motivado el interés por este tipo de producción en los últimos años.

El tamaño promedio del rebaño fue de 36,6 cabezas de ovinos, con valores mínimos de 3 y máximos de 400 , lo que refuerza la percepción de que la actividad ovina es aceptada por un amplio rango de productores a diferentes escalas, pasando desde la cría eventual en instalaciones improvisadas, hasta la establecida en unidades con infraestructura especializada. Las razas empleadas para sementales fueron: Dorper, Pelibuey, Katahdin, Black Belly y otros, con valores de $36,36,29,2,11,2$ y 4,3\%, respectivamente, y para vientres los genotipos empleados fueron: Pelibuey, Pelibuey x Katahdin, Black Belly, Pelibuey x Dorper, Dorper x Katahdin, Black Belly x Dorper y otros, con proporciones de 42,6, 17,4, 13,4, 10,6, 9,9,
3,7 y $3,7 \%$ respectivamente. Estos datos coinciden con lo reportado para el estado de Yucatán por Góngora et al. (2010), quienes mencionan que las razas más empleadas son Pelibuey $(45,1 \%)$, Dorper (35\%), Katahdin $(13,9 \%)$ y Black Belly $(5,9 \%)$; para el país, AMCO (2007) indica que las principales razas de ovinos utilizadas son: Rambouillet, Dorset, Hampshire, Suffolk, Katahdin, Pelibuey, Black Belly, Saint Croix y Dorper.

En el análisis general de los datos se encontró correlación negativa entre la edad (años) y la escolaridad (años) $(r=-0,493 ; \mathrm{P}<0,05)$, a más edad de los responsables de los agroecosistemas se observó una menor escolaridad, y que en promedio fue de 5,7 años; el bajo índice de escolaridad es un fenómeno recurrente en el medio rural de México (Ordaz, 2009) y Latinoamérica en general (CEPAL, 2012). Los resultados de escolaridad encontrados en el presente estudio difieren con los reportados para sistemas de producción ovina en Cuba en donde se encontró que la escolaridad predominante de los productores fue de noveno grado (educación secundaria o técnica) (Borroto et al., 2011).

La residencia del productor en el agroecosistema se correlacionó positivamente con la extensión (ha) de la unidad de producción $(\mathrm{r}=0,460 ; \mathrm{P}<0,05)$. Del total de productores clasificados con residencia en la unidad de producción, el $69 \%$ presentó ovinocultura de traspatio con áreas menores a dos hectáreas; el $8 \%$ disponía de siete hectáreas en promedio y el $23 \%$ presentó una media de 43,5 hectáreas en producción.

Dentro de los tres tipos de mano de obra que se manejaban en los agroecosistemas se encontró correlación negativa entre la mano de obra familiar con contratada fija $(\mathrm{r}=-0,493 ; \mathrm{P}<0,05)$, y la contratada eventual $(\mathrm{r}=-0,477 ; \mathrm{P}<0,05)$. A medida que hubo mayor disponibilidad y participación de la mano de obra familiar disminuyó la dependencia de la asalariada.

La extensión del agroecosistema (ha) se correlacionó positivamente con el tamaño del rebaño (cabezas) $(\mathrm{r}=0,593 ; \mathrm{P}<0,05)$, lo cual resulta lógico, ya que una mayor disponibilidad de tierras incrementa la capacidad de mantener una mayor proporción de ganado, debido a que se dispone de mayor cantidad de recurso forrajero o en su caso de monte (sucesión secundaria) en donde acostumbra pastorear el ganado, principalmente en la época seca, cuando las gramíneas disminuyen la producción de biomasa y especies 
arbóreas y arbustivas representan una de las principales alternativas nutricionales.

El tamaño del rebaño mostró correlación positiva con el tipo de comercialización de sus productos (unidad o lote) $(\mathrm{r}=0,509 ; \mathrm{P}<0,05)$, resultando que los sistemas con rebaños con más de quince animales tenían mayores oportunidades de comercializar los ovinos en lotes más de dos veces al año, mientras que los sistemas con rebaños de menor tamaño se limitan a la venta ocasional de manera individual.

La organización entre ovinocultores es deficiente, se encontró que el $26 \%$ de los productores pertenece a una organización especializada en ovinos, el $6 \%$ pertenece a productores de bovinos regional o local y el $68 \%$ no pertenece a alguna organización de productores. Estos datos muestran la importancia de trabajar en los aspectos relacionados con la organización de productores si se desea desarrollar la ganadería ovina en el estado de Yucatán.

\section{Características de los agroecosistemas con ovinos del oriente de Yucatán}

En el análisis de conglomerados se detectaron cuatro grupos $(\mathrm{G})$ diferentes de agroecosistemas, se denominaron A, B, C y D; de los cuales GA=sistemas de producción con responsables de edad avanzada, escolaridad media, alta cantidad de tierras, rebaños grandes y más años de practicar la ovinocultura, $\mathrm{GB}=$ sistemas de producción con responsables de edad intermedia, mayor escolaridad, cantidad intermedia de tierras y tamaño intermedio de rebaños, $\mathrm{GC}=$ sistemas de producción con responsables de edad avanzada, menor escolaridad, baja cantidad de tierras y reducido tamaño de rebaños, y GD= sistemas con los responsables más jóvenes, escolaridad alta, baja cantidad de tierras, los rebaños más pequeños y menor tiempo de practicar la ovinocultura.

El GD presentó el menor promedio de edad (41,6 años) (Cuadro 2). Del total de los responsables

Cuadro 2. Características descriptivas de los grupos de agroecosistemas con ovinos en la región oriente de Yucatán, México. 2013.

Table 2. Descriptive characteristics of the groups of agroecosystems with sheep in the eastern side of Yucatan, Mexico. 2013.

\begin{tabular}{lcccc}
\hline Variable & GA $(\mathbf{n = 9})$ & GB $(\mathbf{n = 2 7})$ & GC $(\mathbf{n = 5 2})$ & GD $(\mathbf{n = 7 3})$ \\
\hline Edad del responsable (años) & $61,1 \pm 14,0 \mathrm{a}$ & $51,2 \pm 12,5 \mathrm{ab}$ & $61,7 \pm 10,2 \mathrm{a}$ & $41,6 \pm 11,3 \mathrm{~b}$ \\
Escolaridad del responsable (años) & $5,3 \pm 4,6 \mathrm{ab}$ & $9,5 \pm 5,8 \mathrm{a}$ & $3,0 \pm 2,0 \mathrm{~b}$ & $6,3 \pm 3,8 \mathrm{a}$ \\
Extensión (has) & $216,5 \pm 98,5 \mathrm{a}$ & $60,4 \pm 36,3 \mathrm{~b}$ & $22,9 \pm 15,4 \mathrm{~b}$ & $7,8 \pm 12,1 \mathrm{~b}$ \\
Tamaño del rebaño (cabezas) & $105,2 \pm 123,7 \mathrm{a}$ & $79,1 \pm 42,0 \mathrm{ab}$ & $26,1 \pm 18,8 \mathrm{~b}$ & $19,9 \pm 15,2 \mathrm{~b}$ \\
Tiempo en la ovinocultura (años) & $15,3 \pm 14,1$ & $11,0 \pm 7,3$ & $11,0 \pm 10,0$ & $7,1 \pm 6,1$ \\
Organizados (\%) & $44,5 \mathrm{a}$ & $33,3 \mathrm{ab}$ & $25,0 \mathrm{~b}$ & $23,3 \mathrm{~b}$ \\
Acceso al financiamiento (\%) & 0 & $7,4 \mathrm{a}$ & $1,9 \mathrm{~b}$ & $1,4 \mathrm{~b}$ \\
Acceso a apoyos gubernamentales (\%) & $55,5 \mathrm{a}$ & $59,2 \mathrm{a}$ & $32,7 \mathrm{a}$ & $16,4 \mathrm{~b}$ \\
Asistencia técnica (\%) & 0 & 3,7 & 0 & 0 \\
\hline
\end{tabular}

Literales diferentes en la misma fila indican diferencia estadística Tukey $(\mathrm{P}<0,05)$ / Different literals in the same row indicate statistical difference Tukey $(\mathrm{P}<0.05)$.

$\mathrm{GA}=$ responsables de edad avanzada, escolaridad media, alta cantidad de tierras, rebaños grandes y más años de practicar la ovinocultura (elderly people in charge, middle schooling and high amount of land, large herds or more years practing sheep farming), $\mathrm{GB}=\mathrm{responsables}$ de edad intermedia, mayor escolaridad, cantidad intermedia de tierras y tamaño intermedio de rebaños (middle age people in charge, higher schooling and intermediate amount of land, intermediate herd size), GC= responsables de edad avanzada, menor escolaridad, baja cantidad de tierras y reducido tamaño de rebaños (elderly people in charge, less schooling, low amount of land and reduced herd size), y GD= responsables más jóvenes, escolaridad alta, baja cantidad de tierras, los rebaños más pequeños y menor tiempo de practicar la ovinocultura (younger people in charge, high schooling, low amount of land, small herd size, and less time performing the activity). 
de los agroecosistemas con ovinos en el oriente de Yucatán el 3, 11, 17, 18, 26, 19 y 6\% se encontraban en los siguientes rangos de edad: menos de 20, 20 a 30,31 a 40,41 a 50,51 a 60,61 a 70 y más de 70 años respectivamente, en donde fue posible observar que el $61 \%$ de los productores se encuentra en edad productiva de 30 a 60 años; además, que el 14\% estuvo representado por productores menores a 30 años y el $25 \%$ por productores con más de 60 años. Por tanto, puede considerarse que la edad de los productores ganaderos del oriente de Yucatán no es un factor que determine la adopción de la ovinocultura como actividad productiva, dado que se encuentra presente en diferentes rangos. Puede considerarse a la ovinocultura como una estrategia admitida para el manejo del riesgo, puesto que ha iniciado como una actividad secundaria en todos los casos.

Se presentaron dos tipos de tenencia de la propiedad de tierra entre grupos, la privada y la ejidal, en el GA el $89 \%$ tiene propiedad privada y el $11 \%$ ejidal; mientras que para los grupos B, C y D, la propiedad privada representó el 55,5, 25 y 72,6\%, respectivamente.

El pastoreo de los ovinos representó una carga animal (CA) baja en los cuatro grupos de AES, con valores de 0,43 (GA), 0,11 (GB), 0,10 (GC) y 0,22 (GD) UA/ha, considerando $\mathrm{UA}=450 \mathrm{kgPV}$. Sin embargo, en dichas áreas suele presentarse simultáneamente pastoreo de bovinos de manera ocasional o permanente, con CA variables.

\section{Infraestructura, financiamiento y consultoría}

En relación a la infraestructura, solamente se observó diferencias en el corral de manejo ( $\mathrm{p}=0,00869)$, ocupando el primer lugar en los AES para los cuatro grupos (Figura 1). El corral de maternidad fue la infraestructura menos empleada en el total de los agroecosistemas. Resultados similares fueron reportados por Valerio et al. (2010) en República Dominicana, en donde encontraron un promedio de 2,3 corrales de manejo por explotación, y que se carecía de instalaciones de mayor especificidad, como áreas para manejo de las crías. La infraestructura que poseen los ovinocultores de la región oriente de Yucatán es mayor a la reportada por Borroto et al. (2011) en Cuba, quienes mencionaron que el $80 \%$ de los sistemas evaluados presentó una infraestructura mínima de tipo rústico

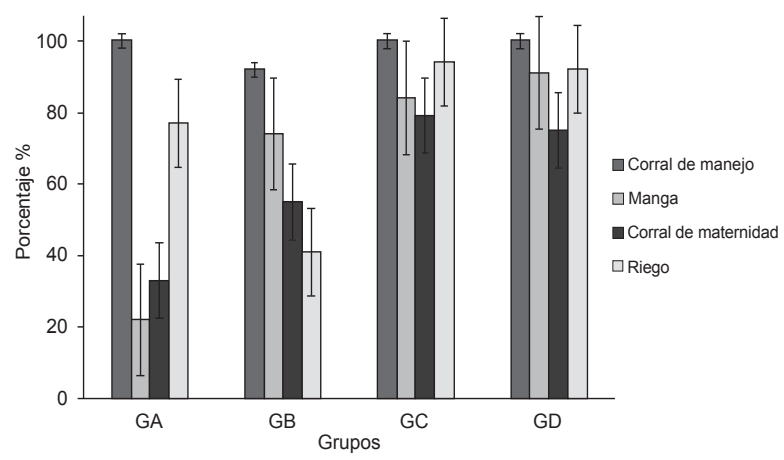

Figura 1. Infraestructura especializada en agroecosistemas con ovinos de la región oriente de Yucatán, México. 2013.

Figure 1. Specialized infrastructure in agroecosystems with sheep of the eastern side of Yucatan, Mexico. 2013.

$\mathrm{GA}=$ responsables de edad avanzada, escolaridad media, alta cantidad de tierras, rebaños grandes y más años de practicar la ovinocultura (elderly people in charge, middle schooling and high amount of land, large herds or more years practing sheep farming), $\mathrm{GB}=$ responsables de edad intermedia, mayor escolaridad, cantidad intermedia de tierras y tamaño intermedio de rebaños (middle age people in charge, higher schooling and intermediate amount of land, intermediate herd size), GC= responsables de edad avanzada, menor escolaridad, baja cantidad de tierras y reducido tamaño de rebaños (elderly people in charge, less schooling, low amount of land and reduced herd size), y GD= responsables más jóvenes, escolaridad alta, baja cantidad de tierras, los rebaños más pequeños y menor tiempo de practicar la ovinocultura (younger people in charge, high schooling, low amount of land, small herd size, and less time performing the activity).

que permitía proteger al rebaño del sol y las lluvias. El hecho de que el corral de manejo ocupara el primer lugar se debe a que el abigeato es un problema presente en las regiones ganaderas del trópico, y por ende, los productores tratan de mantener seguro su rebaño resguardándolo en las noches (Rodríguez, 2014).

Respecto al acceso a créditos financieros, el GB obtuvo la mayor cantidad de financiamientos ejercidos, en este grupo el 7,4\% de los productores había accedido a algún crédito, mientras que en GA ningún agroecosistema ha adquirido créditos; este comportamiento se relaciona al hecho que GA tuvo más acceso a apoyos gubernamentales $(55,5 \%)$, lo que disminuyó la necesidad de contratar créditos, hacia los que existe cierto rechazo al considerarlos caros. La falta de inversión de capital económico en la actividad ovina de la región, mediante los diferentes 
esquemas de financiamiento, propicia que el desarrollo tecnológico y productivo sean lentos y diferenciados entre los diferentes tipos de agroecosistemas.

Los servicios de consultoría son mínimos, solo el 3,7\% de los productores del GB manifestó haber recibido algún tipo de capacitación o asistencia técnica. En este sentido, el 87,6\% de los responsables de los agroecosistemas manifestó estar interesado en recibir capacitación sobre el manejo del ganado ovino; sin embargo, dijeron desconocer las instituciones que brindan dicho servicio y la gestión del mismo. Productores de diferentes grupos coincidieron en mencionar que la actividad ovina requiere del apoyo de diferentes instancias para proporcionar asistencia técnica, también consideraron necesaria la oferta de créditos (gubernamentales y no gubernamentales), así como el diseño de programas específicos que consideren las particularidades de cada grupo de productores por parte de la Secretaria de Desarrollo Rural del Estado. Se requiere fomentar la organización y mejorar la capacitación en aspectos como: manejo sanitario, nutrición, reproducción, manejo de praderas y mejoramiento genético.

\section{Mano de obra}

La mano de obra utilizada en los agroecosistemas de la región oriente de Yucatán tiene diferentes orígenes: familiar, contratada fija y contratada eventual. La mano de obra familiar estuvo presente de manera importante en los cuatro grupos $(66,85,100$ y $96 \%$ para el grupo A, B, C y D), respectivamente, estos valores fueron similares a los reportados en sistemas de producción de ovinos del estado de Veracruz, México, donde encontró que la mano de obra familiar representó el 93, 71 y 82\% en sistemas de subsistencia, transición y comerciales, respectivamente (Pérez et al., 2011). La mano de obra contratada fija fue del $55 \%$ en GA, 44 en GB, $0 \%$ en GC y $82 \%$ en GD, mientras que en el mismo orden, la mano de obra contratada eventual fue de $44,52,7,6$ y $11 \%$. De acuerdo a estos resultados, los agroecosistemas de la región oriente de Yucatán mostraron alta dependencia de la mano de obra generada en el seno de la familia. Este fenómeno no se ajusta con lo reportado por Giorgis et al. (2011), quienes mencionaron que al intensificarse la producción en los sistemas ganaderos, la dependencia de la mano de obra familiar disminuye, incrementándose la mano de obra asalariada. Esta diferencia se debe a que en la región estudiada, en la mayoría de los agroecosistemas, la ovinocultura se encuentra asociada a otras prácticas productivas que propician la contratación fija o eventual de jornales, los cuales se ocupan secundariamente del manejo del rebaño, y en los agroecosistemas que la ovinocultura constituye la primera opción productiva, no presenta un manejo intensivo.

\section{Manejo reproductivo}

El empadre controlado fue el método de reproducción más empleado y estuvo presente en 77, 70, 84 y $73 \%$ en los GA, GB, GC y GD, respectivamente, manejando en promedio 1,89 empadres al año (Cuadro 3). La relación de machos por hembra fue más baja en GA $(0,044)$ y GB $(0,047)$, lo que equivale a un semental por cada veintiún vientres. En GD se observó la relación más alta con un valor promedio de 0,165 , lo que equivale a un semental por cada seis vientres. Estos datos indican un subaprovechamiento de los sementales, dado que se ha establecido que una relación de 0,333 (un semental por cada treinta hembras) puede ser empleada en sistemas de producción ovina (Berumen, 2004). Los resultados aquí encontrados son superiores a los reportados por Valerio et al. (2010) en República Dominicana, en donde encontraron una relación promedio de 0,027 M:H, lo que representó un semental por cada 36 vientres. Al existir una sobrepoblación de sementales se incrementan los costos de producción, y el manejo de los empadres tiende a ser impráctico por la carencia de infraestructura.

\section{Nutrición de ovinos y manejo de praderas}

En los cuatro grupos, se encontró que la alimentación de los ovinos en los AES, se basó principalmente en pastoreo de potreros con gramíneas mejoradas y con encierros por la noche con un $78,8 \%$; seguido de manejo semi-estabulado $(11,1 \%)$ y el manejo estabulado (9,7\%); de estos el 4,2\% manejó este tipo de alimentación debido a la falta de potreros (Figura 2). Estos resultados coinciden con los reportados por Valerio et al. (2010), quienes encontraron que el $94 \%$ de las explotaciones manejan el pastoreo continuo en praderas de gramíneas. Estas, en general, no recibían fertilización y los periodos de ocupación no se establecían de acuerdo a la capacidad 
Cuadro 3. Manejo reproductivo en los agroecosistemas con ovinos de la región oriente de Yucatán, México, 2013.

Table 3. Reproductive managing in the agroecosystems with sheep of the eastern side of Yucatan, Mexico. 2013.

\begin{tabular}{llll}
\hline Grupos & n & $\begin{array}{c}\text { Relación } \\
\text { macho:hembra }\end{array}$ & $\begin{array}{c}\text { Empadres al } \\
\text { año }\end{array}$ \\
\hline GA & 9 & $0,044 \pm 0,0593 \mathrm{a}$ & $1,888 \pm 0,3118$ \\
GB & 27 & $0,047 \pm 0,0342 \mathrm{a}$ & $1,881 \pm 0,1800$ \\
GC & 52 & $0,140 \pm 0,0247 \mathrm{~b}$ & $2,076 \pm 0,1297$ \\
GD & 73 & $0,165 \pm 0,0208 \mathrm{~b}$ & $1,754 \pm 0,1095$ \\
D.E. & & 0,1825 & 0,9374 \\
E.E & & 0,0143 & 0,1912 \\
\hline
\end{tabular}

Literales diferentes en la misma columna indican diferencia significativa según Tukey $(\mathrm{P}<0,05) /$ Different literals in the same row indicate statistical difference Tukey $(\mathrm{P}<0.05)$.

$\mathrm{GA}=$ responsables de edad avanzada, escolaridad media, alta cantidad de tierras, rebaños grandes y más años de practicar la ovinocultura (elderly people in charge, middle schooling and high amount of land, large herds or more years practing sheep farming), $\mathrm{GB}=$ responsables de edad intermedia, mayor escolaridad, cantidad intermedia de tierras y tamaño intermedio de rebaños (middle age people in charge, higher schooling and intermediate amount of land, intermediate herd size), GC= responsables de edad avanzada, menor escolaridad, baja cantidad de tierras y reducido tamaño de rebaños (elderly people in charge, less schooling, low amount of land and reduced herd size), y GD= responsables más jóvenes, escolaridad alta, baja cantidad de tierras, los rebaños más pequeños y menor tiempo de practicar la ovinocultura (younger people in charge, high schooling, low amount of land, small herd size, and less time performing the activity).

D.E.= desviación estándar, E.E= error estándar / D.E Standar Desviation, E.E. Standar Error.

productiva de las mismas, lo que afectó los índices de ganancia de peso diario de los ovinos.

El sistema de alimentación basado en pastoreo, fue el más usado en los cuatro grupos GA, GB, GC y GD $(p=0,0343)$. Generalmente, se encerraban los animales por la tarde y se les proporcionaba algún suplemento que solía variar en el transcurso del año, en función de los recursos disponibles (granos, pasto de corte, follaje de árboles, esquilmos agrícolas o subproductos de origen animal). El sistema de estabulación completa fue empleado en el 11, 7,5, 9,6 y $11 \%$ de los agroecosistemas del GB.

El suministro de sal mineral y la aplicación de hierro fueron prácticas comunes en los cuatro grupos,

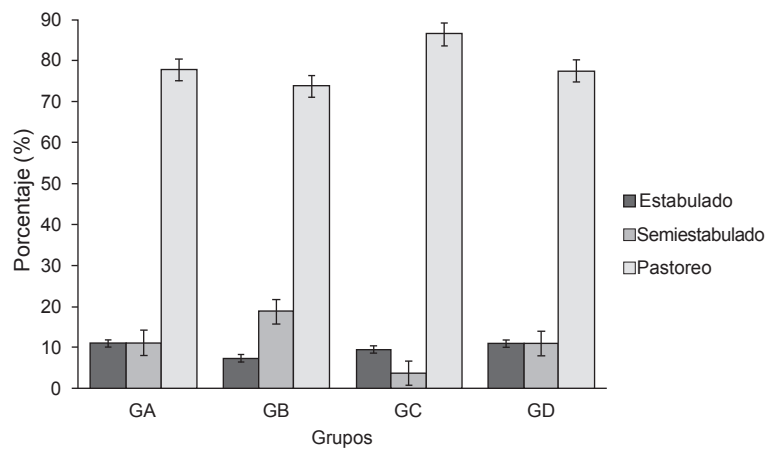

Figura 2. Manejo de alimentación de ovinos en los agroecosistemas de la región oriente de Yucatán, México. 2013.

Figure 2. Sheep feeding handling in the agroecosystems with sheep of the region eastern side of Yucatan, Mexico. 2013.

$\mathrm{GA}=$ responsables de edad avanzada, escolaridad media, alta cantidad de tierras, rebaños grandes y más años de practicar la ovinocultura (elderly people in charge, middle schooling and high amount of land, large herds or more years practing sheep farming), $\mathrm{GB}=$ responsables de edad intermedia, mayor escolaridad, cantidad intermedia de tierras y tamaño intermedio de rebaños (middle age people in charge, higher schooling and intermediate amount of land, intermediate herd size), GC= responsables de edad avanzada, menor escolaridad, baja cantidad de tierras y reducido tamaño de rebaños (elderly people in charge, less schooling, low amount of land and reduced herd size), y GD= responsables más jóvenes, escolaridad alta, baja cantidad de tierras, los rebaños más pequeños y menor tiempo de practicar la ovinocultura (younger people in charge, high schooling, low amount of land, small herd size, and less time performing the activity).

siendo mayor su uso en el GA con 78\%. El empleo de este tipo de suplementación se debió a que son imitadas de la ganadería bovina, la cual es una práctica común, debido a las deficiencias de minerales reportadas en los forrajes de Yucatán (Segura y Castellanos, 1999; Cabrera et al., 2009).

El $100 \%$ de GA, el $74 \%$ de GB, el $90 \%$ de GC y $88 \%$ de GD utilizan algún follaje de arbóreas para alimentar a los ovinos, siendo las especies más usadas (Brosimum alicastrun, Guazuma ulmifolia, Leucaena leucocephala, Piscidia piscipula, Bursera simaruba, Spondias purpurea y Melicoccus olivaeformis), las cuales se encontraron presentes en las unidades de producción en las fracciones de vegetación secundaria que conservaron, aunque también las cosecharon de áreas públicas o de uso común. Con respecto a la disponibilidad de establecer 
sistemas silvopastoriles, el 100, 92, 90 y $79 \%$ de los responsables de los agroecosistemas de GA, GB, GC y GD, respectivamente, mostraron disponibilidad para adoptar la tecnología. Motivados por observaciones directas de la preferencia de los ovinos por el ramoneo de arbóreas y por los efectos positivos observados en épocas de estiaje cuando el forraje de gramínea ha sido escaso y el follaje de árboles representó la única fuente de alimentación. Esta información muestra que es posible implementar programas de establecimiento de sistemas agrosilvopastoriles con los productores con base en el uso de recursos locales para lograr una ovinocultura más amigable con el medio ambiente.

\section{Manejo sanitario}

Según comentarios emitidos por los productores entrevistados, la parasitosis gastrointestinal fue la enfermedad más recurrente en los agroecosistemas de los cuatro grupos, seguida de enfermedades respiratorias (Cuadro 4). Al respecto Rodríguez-Vivas et al. (2001) encontraron que el 95,4\% de las muestras provenientes de ovinos del estado de Yucatán fueron positivas a parásitos gastrointestinales. Para hacer frente a este problema, los ovinocultores han recurrido a desparasitaciones continuas e indiscriminadas con productos químicos, al respecto es necesario el empleo de técnicas alternativas para su control como puede ser manejo del pastoreo (Eysker et al., 2005), uso de arbóreas y arbustivas con presencia de compuestos secundarios (Moreno et al., 2010) y desparasitación dirigida mediante diagnósticos previos (Arece y López, 2013), entre otras.

Debido a que la parasitosis fue la enfermedad más afectiva sobre los ovinos en los agroecosistemas evaluados, la desparasitación contra nematodos gastrointestinales resultó ser la práctica sanitaria más empleada, con el $96,8 \%$ del total; mientras que la aplicación de vacunas se realizó en el $71,4 \%$ de los agroecosistemas (Cuadro 5). Se emplearon criterios variados para decidir el momento de la aplicación del tratamiento antihelmíntico. El criterio más empleado fue el manejo de un calendario fijo de desparasitación y los periodos entre aplicaciones fueron de $1,2,3,4$, 6 y 12 meses con $32,15,30,0,75,14$ y $1 \%$ promedio de los cuatro grupos. En segundo lugar, se ubicó el

Cuadro 4. Principales enfermedades de los ovinos en los agroecosistemas de la región oriente de Yucatán, México. 2013.

Table 4. Main diseases of sheep in the agroecosystems of the eastern side of Yucatan, Mexico. 2013 .

\begin{tabular}{lccccc}
\hline & & \multicolumn{4}{c}{ Enfermedad (\%) } \\
\cline { 3 - 6 } Grupo & N & Parasitosis & Respiratoria & Otra & Ninguna \\
\hline GA & 9 & 100 & 0 & 0 & 0 \\
GB & 27 & 66,0 & 18,5 & 7,4 & 7,4 \\
GC & 52 & 61,5 & 19,2 & 13,4 & 5,7 \\
GD & 73 & 58.9 & 23,2 & 6,8 & 11,0 \\
$\bar{x}$ & & 71,6 & 15,2 & 6,9 & 6,0 \\
\hline
\end{tabular}

$\mathrm{GA}=$ responsables de edad avanzada, escolaridad media, alta cantidad de tierras, rebaños grandes y más años de practicar la ovinocultura (elderly people in charge, middle schooling and high amount of land, large herds or more years practing sheep farming), GB= responsables de edad intermedia, mayor escolaridad, cantidad intermedia de tierras y tamaño intermedio de rebaños (middle age people in charge, higher schooling and intermediate amount of land, intermediate herd size), GC= responsables de edad avanzada, menor escolaridad, baja cantidad de tierras y reducido tamaño de rebaños (elderly people in charge, less schooling, low amount of land and reduced herd size), y GD= responsables más jóvenes, escolaridad alta, baja cantidad de tierras, los rebaños más pequeños y menor tiempo de practicar la ovinocultura (younger people in charge, high schooling, low amount of land, small herd size, and less time performing the activity). 
Cuadro 5. Prácticas sanitarias en los agroecosistemas con ovinos de la región oriente de Yucatán, México. 2013.

Table 5. Sanitary practices of the sheep ones in the agroecosystems of the region eastern side of Yucatan, Mexico. 2013.

\begin{tabular}{lccccc}
\hline Práctica & GA & GB & GC & GD & $\overline{\mathbf{x}}$ \\
\hline Aplica vacunas & 100 & 81,5 & 73,0 & 63,0 & 79,4 \\
Desparasita & 100 & 100 & 96,0 & 96,0 & 98,0 \\
Criterio para desparasitar & & & & & \\
Análisis de laboratorio & 11,0 & 7,5 & 0 & 0 & 4,6 \\
Calendario & 44,0 & 74,0 & 64,0 & 54,3 & 59,0 \\
Síntomas & 33,0 & 18,5 & 32,0 & 45,7 & 32,3 \\
Calendario + síntomas & 11,0 & 0 & 4,0 & 0 & 3,7 \\
\hline
\end{tabular}

$\mathrm{GA}=$ responsables de edad avanzada, escolaridad media, alta cantidad de tierras, rebaños grandes y más años de practicar la ovinocultura (elderly people in charge, middle schooling and high amount of land, large herds or more years practing sheep farming), $\mathrm{GB}=$ responsables de edad intermedia, mayor escolaridad, cantidad intermedia de tierras y tamaño intermedio de rebaños (middle age people in charge, higher schooling and intermediate amount of land, intermediate herd size), GC= responsables de edad avanzada, menor escolaridad, baja cantidad de tierras y reducido tamaño de rebaños (elderly people in charge, less schooling, low amount of land and reduced herd size), y GD= responsables más jóvenes, escolaridad alta, baja cantidad de tierras, los rebaños más pequeños y menor tiempo de practicar la ovinocultura (younger people in charge, high schooling, low amount of land, small herd size, and less time performing the activity).

criterio de aplicar el tratamiento cuando se observaron síntomas en los ovinos, como pérdida de peso, de apetito, decaimiento y presencia de mucosidad nasal.

\section{Comercialización de los productos ovinos}

El principal producto de la ovinocultura fueron los corderos pre-finalizados en pie con pesos que oscilaban entre 35 y $40 \mathrm{~kg}$, lo que representó el 78, 66,72 y $81 \%$ en los agroecosistemas de GA, GB, GC y GD, respectivamente; el 11 (GA), 33 (GB), 21 (GC) y $9,5 \%$ (GD), mencionó que, además de corderos en pie, ofertaban otros productos como sementales, vientres o cortes. El resto de los productores mencionó que se especializaba en productos como sementales, vientres y cortes, aunque, en porcentajes mínimos y ocasionalmente, vendían corderos destetados. En la
Figura 3 se ejemplifica el destino de las ventas en los cuatro grupos de AES, destacando que el GA vendía más de la mitad de sus productos a intermediarios, por su parte el GD vendía el mayor porcentaje de sus productos a consumidores finales, que se encontraban representados por el público en general que adquiere ovinos en pie para preparar en festividades, esta situación coincide con el hecho que fue el grupo con los rebaños más pequeños y menor disponibilidad de tierras, en traspatios (Cuadro 2), por lo que sus borregos se encontraban en áreas urbanas o periurbanas y fueron más accesibles a la población de la comunidad. Los precios de venta solían variar de los $\$ 25.00$ hasta los $\$ 31.00$ por kilogramo, siendo el desconocimiento de los precios de mercado actualizados la principal causa del diferencial de precios de venta. 


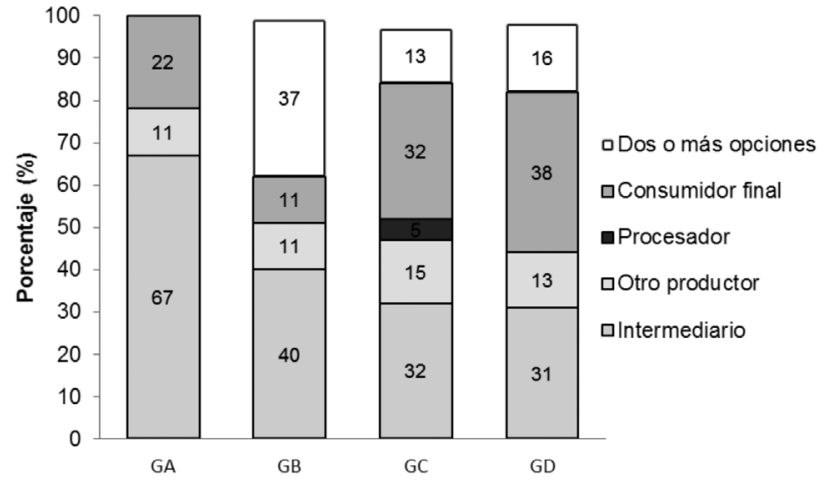

Figura 3. Destino de la venta de productos ovinos de los agroecosistemas de la región oriente de Yucatán, México. 2013.

Figure 3. Destination of the sale of sheep products of the agroecosystems of the eastern side of Yucatan, Mexico. 2013.

$\mathrm{GA}=$ responsables de edad avanzada, escolaridad media, alta cantidad de tierras, rebaños grandes y más años de practicar la ovinocultura (elderly people in charge, middle schooling and high amount of land, large herds or more years practing sheep farming), $\mathrm{GB}=$ responsables de edad intermedia, mayor escolaridad, cantidad intermedia de tierras y tamaño intermedio de rebaños (middle age people in charge, higher schooling and intermediate amount of land, intermediate herd size), $\mathrm{GC}=$ responsables de edad avanzada, menor escolaridad, baja cantidad de tierras y reducido tamaño de rebaños (elderly people in charge, less schooling, low amount of land and reduced herd size), y GD= responsables más jóvenes, escolaridad alta, baja cantidad de tierras, los rebaños más pequeños y menor tiempo de practicar la ovinocultura (younger people in charge, high schooling, low amount of land, small herd size, and less time performing the activity).

\section{LITERATURA CITADA}

AMCO (Asociación Mexicana de Criadores de Ovinos). 2007. Catálogo de razas. http://www.uno.org.mx/ razas_ovinas/catalogo_razas.pdf (consultado 6 feb. 2015).

Arece, J., e Y. López. 2013. Validación del método FAMACHAC en la detección de anemia en ovejas Pelibuey en Cuba. Rev. Pastos y Forrajes 36:479-484.

Arteaga, C.J.D. 2007. Diagnóstico actual de la situación de los ovinos en México. La revista del borrego. http:// www.borrego.com.mx/opinion/diagnostico-actual-dela-situacion-de-los-ovinos-en-mexico/ (consultado 26 feb. 2104).
Berumen, A.A.C. 2004. Manejo reproductivo del rebaño ovino. En: S.D. Hernández, editor, Producción de ovinos en zonas tropicales. Colegio de Postgraduados, Fundación Produce Tabasco, A.C. e Instituto para el Desarrollo de Sistemas de Producción del Trópico Húmedo de Tabasco (ISPROTAB). Villahermosa, Tabasco, MEX. p. 105-120.

Borroto, A., C. R. Pérez, C.A. Mazorra, C.A. Pérez, M. Barrabí, y C.A Agueda. 2011. Caracterización socioeconómica y tecnológica de la producción ovina en Ciego de Ávila, región Central de Cuba (Parte I). Rev. Pastos y Forrajes 34:199-210.

Cabrera, T.E.J., R.E.E. Sosa, R.A.F. Castellanos, B.A.O. Gutiérrez, y S.J.H. Ramírez. 2009. Comparación de la concentración mineral en forrajes y suelos de zonas ganaderas del estado de Quintana Roo, México. Vet. Mex. 40:167-179.

CEPAL (Comisión Económica para América Latina y el Caribe). 2012. Panorama Social de América Latina. Documento informativo. http://www.cepal.org/ publicaciones/xml/5/48455/panora masocial2012docirev.pdf (consultado 15 feb. 2014).

Córdova, A.V. 1993. Factores para la adopción de tecnología agrícola en el Plan Chontalpa, Tab., México. Tesis M.C., Colegio de Postgraduados, Campus Puebla, Puebla, MEX.

Eysker, M., N. Bakker, F.N.J. Kooyman, and H.W. Ploeger. 2005. The possibilities and limitations of evasive grazing as a control measure for parasitic gastroenteritis in small ruminants in temperate climates. Vet. Parasitol. 129(1-2):95-104.

FAO (Organización de las Naciones Unidas para la Alimentación). 2013. Carne y productos cárnicos. http://www.fao.org/ag/againfo/themes/es/meat/home. html (consultado 10 ene. 2014)

Frías, J.C., E.M. Aranda, J.A. Ramos, C. Vázquez, y P. Díaz. 2011. Calidad y rendimiento en canal de corderos en pastoreo suplementados con caña de azúcar fermentada. Av. Inv. Agropec. 15(3):33-44.

Gallardo, L.F., D.D. Riestra, S.A. Aluja, y D.J.P. Martínez. 2002. Factores que determinan la diversidad agrícola y los propósitos de producción en los agroecosistemas del municipio de Paso de Ovejas, Veracruz, México. Agrociencia 36:495-502.

Giorgis, A., M.J.M. Perea, M.A. García, C.A.G. Gómez, P.A. Sánchez, y A. Larrea. 2011. Caracterización técnico-económica y tipológica de las explotaciones 
lecheras de la Pampa (Argentina). Rev. Científ. FCVLUZ 21:340-352.

Góngora-Pérez, R.D., S.F. Góngora-González, M.A. Magaña-Magaña, y L.P.E. Lara. 2010. Caracterización técnica y socioeconómica de la producción de ovina en el estado de Yucatán, México. Agron. Mesoam. 21:131-144.

INEGI (Instituto Nacional de Estadística y Geografía). 2013. Censo agrícola ganadero y forestal 2007. http://www. inegi.org.mx/est/contenidos/proyectos/Agro/ca2007/ Resultados_Agricola/default.aspx (consultado $21 \mathrm{feb}$. 2014)

Martínez-Partida, J.A., L. Jiménez-Sánchez, J.G. HerreraHaro, E. Valtierra-Pacheco, E. Sánchez-López, y M.C. López-Reyna. 2011. Ganadería ovino-caprina en el marco del programa de desarrollo rural en Baja California. Univ. Cienc. 27:331-344.

Morales, M.M., D.J. Martínez, H.G. Torres, y V.J.E Pacheco. 2004. Evaluación del potencial para la producción ovina con el enfoque de agroecosistemas en un ejido de Veracruz, México. Rev. Tec. Pec. Mex. 42:347-359.

Moreno, F.C., I.J. Gordon, A.D. Wright, M.A. Benvenutti, y C.A. Saumell. 2010. Efecto antihelmíntico in vitro de extractos de plantas sobre larvas infectantes de nematodos gastrointestinales de rumiantes. Arc. Med. Vet. 42:155-163.

Ojeda. M. 1999. Análisis exploratorio de datos con énfasis multivariado en el contexto de aplicaciones ecológicas. Universidad Veracruzana, MEX.

Ordaz, D.J.L. 2009. México: impacto de la educación en la pobreza rural. En: Serie estudios y perspectivas No. 105. Naciones Unidas, CEPAL. Sede Subregional, México. México, D.F. http://www.cepal.org/ publicaciones/xml/4/35044/serie_105.pdf (consultado 10 abr. 2014).

Partida, P.J.A., V.D. Braña, S.H. Jiménez, R.F.G. Ríos, y R.G. Buendía. 2013. Producción de carne ovina. Libro técnico. Centro Nacional de Investigación Disciplinaria en Fisiología y Mejoramiento Animal. Ajuchitlan, Querétaro, MEX.

Pérez, H.P., J. Vilaboa, H. Chalate, B. Candelaria, P. Díaz, y S. López. 2011. Análisis descriptivo de los sistemas de producción con ovinos en el estado de Veracruz, México. Rev. Científ. FCV-LUZ 21:327-334.

Rodríguez, D.J.L. 2014. Pobreza, hambre y abigeato: otras formas de resistencia en Sacatepéquez decimonónico. Rev. Pueblos y Fronteras Digital 9(17):127-132.
Rodríguez-Vivas, R.I., L.A. Cob-Galera, y J.L. DomínguezAlpizar. 2001.Frecuenciadeparásitos gastrointestinales en animales domésticos diagnosticados en Yucatán, México. Rev. Biomed. 12:19-25.

Santesmases, M.M. 2005. Diseño y análisis de encuestas en investigación social y de mercados. Ediciones Pirámide, Madrid, ESP.

Sañudo, A.C. 2008. Calidad de la canal y de la carne ovina y caprina y los gustos de los consumidores. Rev. Bras. Zootec. 37:143-160.

Segura, C.V.M., y R.A.F. Castellanos. 1999. Efecto de la suplementación fosforada sobre la ganancia de peso de bovinos en pastoreo en Yucatán, México. Vet. Mex. 30:257-261.

SIAP (Servicio de Información Agroalimentaria y Pesquera). 2012. Resumen nacional (producción, precio, valor, animales sacrificados y peso. http://www.siap.gob. mx/resumen -nacional-pecuario/ (consultado 18 feb. 2014).

SIAP (Servicio de Información Agroalimentaria y Pesquera). 2013. Resumen nacional (producción, precio, valor, animales sacrificados y peso. http://www.siap.gob. mx/resumen -nacional-pecuario/ (consultado 18 feb. 2014).

SIAP (Servicio de Información Agroalimentaria y Pesquera). 2014. Resumen nacional (producción, precio, valor, animales sacrificados y peso. http://www.siap.gob. $\mathrm{mx} /$ resumen -nacional-pecuario/ (consultado 18 feb. 2014).

SPSS Statistics. 2006. Base 17.0. SPSS Inc. Chicago, IL, USA. http://www.spss.com (consultado 10 feb. 2014).

Suárez, H., G. Aranda, y J.M. Palma. 2012. Propuesta para la adopción de tecnología en el sistema bovino de doble propósito. Av. Inv. Agropec. 16(3):83-91.

Valerio, D., A. García, R. Acero, J. Perea, M. Tapia, y M. Romero. 2010. Caracterización estructural del sistema ovino-caprino en la región noreste de República Dominicana. Arch. Zootec. 59:333-343.

Vilaboa, J., P. Díaz, R. Ruíz, D. Platas, S. González, y F. Juárez. 2009. Caracterización socioeconómica y tecnológica de los agroecosistemas con ganado bovino de doble propósito en la región del Papaloapan, Veracruz. Trop. Subtrop. Agroecosyt. 10:53-63. 